\title{
The diversity management paradox in globalization - the Swedish IKEA way
}

\author{
Steffen Korsgaard, Morten Rask and Jakob Lauring \\ Department of Management, Aarhus School of Business
}

IKEA, the global business of retail home furniture and house ware, is often used as a prime example of a firm that have had a rapid internationalization process (Hollensen, 2007b). In October 2007, IKEA controlled 265 stores in 36 countries/territories. The IKEA Group itself owns 235 stores in 24 countries. The other 30 stores are owned and run by franchisees outside the IKEA Group in 15 countries/territories (IKEA, 2007b). The IKEA concept and the IKEA trademark are owned by Inter IKEA Systems B.V. in the Netherlands. Inter IKEA Systems B.V. is the franchiser for all IKEA stores, both within and outside the IKEA Group (IKEA, 2007a), which means a franchising contract authored centrally at IKEA controls all business processes in all stores in all countries (Hollensen, 2007b).

IKEA is a king of globalization compared to the many local competitors in the furniture industry (Capell et al., 2005; Hollensen, 2007b), if we understand globalization as a process leading to greater interdependence and mutual awareness among economic, political, and social units in the world, and among actors in general (Guillen, 2001). As such globalization means that big global players like IKEA are exposed to an increased global awareness and interdependence, which is recognised when IKEA promotes the reason for considering IKEA as a future employer. This can be seen at their global website under the title "why work at IKEA?". In the last point of nine, IKEA explains the background for their diversity management practice: "We encourage an environment where people of different views, age, nationality, gender and ethnic background feel welcome. We believe that a diverse workforce will improve business results, strengthen our competitiveness and make IKEA a better place to work" (IKEA, 2006). This reflects the high importance and big effort IKEA puts into recruitment (Björk, 1998; Kling \& Goteman, 2003) and how they related this to the management of human differences as part of the globalization strategy.

On the one hand IKEA has for many years been in the globalization phase where synergy between the different parts of the organization is in focus (Douglas \& Craig, 1989). The HQ called IKEA international was moved from Sweden to Denmark in 1975 and has since 2001 been located in the Netherlands. IKEA has been able to follow a almost pure global strategy (Yip, 1989) since IKEA has a 
significant market share on 5-10\% of the total furnishing market in each of the target countries (Björk, 1998; CapellSainsLindbladPalmerBushRoberts \& Hall, 2005; Hollensen, 2007b), the furniture products are fully standardized in each country while some of the house ware products can be adapted (Hollensen, 2007b; Solomon, 1991; Woods \& Sciarini, 1995), marketing is uniform worldwide (Hollensen, 2007b), and the competitive moves are integrated across countries (Krugman, 1990). The IKEA corporation has managed to do that with a diversified country market strategy with a keen concentration on one segment (Ayal \& Zif, 1979), namely the low to middle income young family (Hollensen, 2007b; Kling \& Goteman, 2003). Another strong reason is the superior global branding, where their brand awareness have a significantly higher value than the size of the firm and sales volume (CapellSainsLindbladPalmerBushRoberts \& Hall, 2005; Hollensen, 2007b; Kling \& Goteman, 2003). Branding together with organizational culture are also important for the recruitment process (Kling \& Goteman, 2003).

On the other hand, IKEA do not only sell furniture and house ware, the firm sell Scandinavian lifestyle (CapellSainsLindbladPalmerBushRoberts \& Hall, 2005; Hollensen, 2007b; Kling \& Goteman, 2003). The globalization phase is often understood as going hand-in-hand with a geocentric managing mindset. However, while IKEA is known as a multicultural organization (Kochan et al., 2002), IKEA has had a strong almost ethnocentric (Perlmutter, 1969) Swedish corporate culture (Kling \& Goteman, 2003; KochanBezrukovaElyJacksonJoshiJehnLeonardLevine \& Thomas, 2002) following a home based internationalization strategy (Krugman, 1990). Every time IKEA had struggled because of accelerated internationalization, cultural management policies have been rolled out (Björk, 1998); and the latest addition is diversity management.

To sum up the characteristics of IKEA you could say that the story of IKEA involves several paradoxes that can be boiled down as earlier said by the Harvard Business School Professor Christopher A. Bartlett as "The great challenge of any organization as it becomes larger and more diverse is how to keep the core founding values alive” (Cited in CapellSainsLindbladPalmerBushRoberts \& Hall, 2005). The CEO of IKEA Anders Dahlvig almost says the same when asked about the most challenging issue in the future: "I think the biggest challenge comes from ourselves: to see if we can benefit from the advantages of being big and yet not be affected by the disadvantages” (Kling \& Goteman, 2003).

Thus will this paper focus on IKEA's efforts to meet the challenges of globalization with diversity management. We will discuss the managerial and theoretical implications in order to understand the effects of utilizing diversity management as means for keeping the core corporate culture key values alive when the organization becomes larger and more diverse.

\section{From ethnocentric cultural management to global management in IKEA}

In the late 1960s IKEA expanded their business to the neighbouring Scandinavian countries Norway and Denmark. When the growth started to stagnate in the 1970ties, the focus was to expand to the German speaking European countries. Surprisingly, the conservative country Switzerland was a success for IKEA even though they 
simply moved the Swedish concept to a new market (Björk, 1998). According to Anders Dahlvig CEO of IKEA, this laid the ground for IKEA's international growth success story: "After that, we basically copied the concept worldwide from the 1970s until today. So the fundamental success criteria are fairly old. Although we have continuously adapted and improved them, basically they have been the same all the time” (Kling \& Goteman, 2003). Where IKEA has been extremely successful in implementing the standardised concept with a low national responsiveness on the product offerings (Hollensen, 2007a; Krugman, 1990), IKEA early recognised that the organizational culture needed to be more adaptive. In relation to IKEA's expansion to Switzerland, Germany and Austria, Ingvar Kamrad, the founder of IKEA had some doubts: "You can ask yourself, is it right of us to come in with our style and way of socializing and not adapt to their culture... but we would not destroy their culture - we would create togetherness. And in general it worked out well” (Björk, 1998). However, after the heavy international expansion in the 1970s, the first cultural revolution at IKEA started. The background was that the costefficiency got out of control and the plan called 'Kraft 80' (Power 80) included internal search, self-criticism and concentration of decision making power. These elements became tools for getting IKEA back to its heritage, and to regain its vitality, creativity and flexibility. As a result of this process, IKEA became conscious about the value of the IKEA corporate culture. The culture became a management tool to motivate and make sure that the people in the big origination stick together. Since then IKEA has launched corporate culture campaigns every five years to keep the culture alive. Today the management of IKEA often points out that IKEA has two legs, the business concept and the corporate culture (Björk, 1998; Kling \& Goteman, 2003). The ideal manager was a Swede that knows the country in question. Anders Moberg that was the CEO of IKEA until 1999, advised the foreign co-workers that wanted to climb up the corporate ladder to learn Swedish: "They will have a different understanding of the culture, atmosphere and attitude". In this line of thought it has often been stated that while the corporate language of the IKEA group is English, the language of the corporate culture is Swedish (Björk, 1998). However, there have been critics of the Swedish male dominated management culture in IKEA and concurrently with the geographic expansion of IKEA, where the differences between the official global and the local IKEA corporate culture has widened and forced IKEA to increase the focus on maintaining the corporate culture (Björk, 1998).

Where simplicity describes the business concept, cost-deficiency and togetherness described the corporate culture. The last keyword that altogether is promoted as the key values is respect. Perhaps, because IKEA had experienced major market entry failures in Japan in the 1980s (Capell, 2006; CapellSainsLindbladPalmerBushRoberts \& Hall, 2005) and how they have solves the big problems in the United States (Björk, 1998; Grol \& Schoch, 1998; Solomon, 1991; Zachary, 2005), respect is important to decrease the relatively ethnocentric management in IKEA and to focus on diversity management as the a management tool.

\section{Diversity management in IKEA}

Diversity management as a concept is an American inspired management tool developed in the early 1980's. To begin with the concept was related to Affirmative Action and Equal Opportunity with regard to weaker minorities on the labour market, but has since developed increasingly also to include an argument of business 
opportunities in managing human difference (Wrench, 2002). Here, the focus is on the combination of competencies that can be achieved through diversity management - a combination of competencies that are believed to lead to competitive advantages within areas such as market intelligence or internationalization of the organization. Furthermore it has been argued that synergies from diverse knowledge resources can create better decision making and more creativity and innovation in the business procedures. Finally, value human diversity can be used in branding the organization and for attracting talented employees of different races and genders etc (Cox \& Blake, 1991; Thomas \& Ely, 1996).

This perspective can also be seen in corporate statements made by IKEA: "We see the diversity issue as a matter of creating a more challenging business atmosphere and of course expanding the recruitment base - including everyone and not just Swedish men. It also gives us a diverse workforce with a lot of positive business possibilities" (Kling \& Goteman, 2003). IKEA understands diversity management in the following way (Fajtová, 2007): "Diversity at IKEA is when people from different backgrounds feel comfortable and confident, when they can apply their perspectives and approaches at work and when they are included at all levels and functions in the organization.”

IKEA argue that human diversity handled by a global HRM policy must be context dependent, especially because of the increased heterogeneity in the in customer base and the potential recruitment population in their target markets, where the characteristics of these markets differs from country to country: "As a global player it is very important for IKEA to be aware of what the world looks like today, and how it will change in the future. The only common thing for all of us is that we are different. If we accept and understand this fact we can start to use this Diversity for the best of ourselves and for IKEA" (Fajtová, 2007). The extending internationalization process of the firm the diversity concept is very important in order to maintain a solid firm to recruit people: "I don't think Swedes are fit to be managers at IKEA because of them being Swedes. However, it has been the strategy in the past, which was abandoned about five years ago, and maybe some people still think that way... Today we have a different situation... There are plenty of transfers between countries today” (Kling \& Goteman, 2003).

Even this short summary of the IKEA version of diversity management demonstrates a somewhat paradoxical state of affairs. On the one hand IKEA seek to embrace and respect the fundamental differences of individuals, while on the other hand a strong unified corporate culture. This triggers the question; if and how individuals with differences in background as race, gender, physical ability, ethnicity, sexual orientation and age all can subscribe to the shared corporate values of IKEA and find it a good place to work?

By taking departure from the above mentioned challenges of globalization this paper discuss the meeting of international management and diversity management. What happens when some of the challenges of managing an international corporation are met using diversity management as a tool? This question has formed the centre of the research presented in this paper. To understand the above mentioned paradox and explore this question a closer look at the diversity management practice(s) of IKEA is imperative. 


\section{Methodology and Data}

In the following we will investigate the practice of Diversity Management in IKEA in order to understand if and how Diversity Management is possible in a global organization that is dominated by a non-negotiable standardized corporate culture by utilizing the key-values as simplicity, cost-efficiency, togetherness and respect as the negotiable factors in the local setting.

The primary data is collected from IKEA's homepages that was re-launched during the summer of 2007 (Datamonitor, 2005), where we had a specific focus on the recruitment marketing pages in the different countries. By taking all countries into consideration we have made sure that as diverse background for the job-seekers in focus is as diverse as possible when it comes to their background in terms of race, gender, physical ability, ethnicity, sexual orientation and age

The following pages show the data that is divided into two tables. Table 1 groups the countries where the IKEA Group owns the stores. Table 2 is the group of countries where the stores are owned by franchisees. In all countries we have analyzed the "IKEA Values" webpage promoted to potential job-seekers. In countries that have more than one language present we have analyzed editions in relation to IKEA statements.

Table 1: Diversity management in countries owned by the IKEA Group

\begin{tabular}{|c|c|c|c|}
\hline Country & $\begin{array}{l}\text { Non-negotiable } \\
\text { differences }\end{array}$ & $\begin{array}{l}\text { Negotiable } \\
\text { differences }\end{array}$ & URL \\
\hline Australia & $\begin{array}{l}\text { Work hard, be } \\
\text { yourself }\end{array}$ & $\begin{array}{l}\text { A corporate culture } \\
\text { based on shared values } \\
\text { Togetherness } \\
\text { Cost-Consciousness } \\
\text { Respect } \\
\text { Simplicity }\end{array}$ & $\begin{array}{l}\text { http://www.ikea.com/ms/en_AU/jobs/join_us/ike } \\
\text { a_values/ }\end{array}$ \\
\hline Austria & $\begin{array}{l}\text { Arbeite hart, sei du } \\
\text { selbst } \\
\text { Unsere }\end{array}$ & $\begin{array}{l}\text { Unternehmenskultur } \\
\text { basiert auf geteilten } \\
\text { Werten } \\
\text { Gemeinsamkeit } \\
\text { Kostenbewusstsein } \\
\text { Respektvoller Umgang } \\
\text { Miteinander } \\
\text { Einfachheit } \\
\text { Offenheit }\end{array}$ & $\begin{array}{l}\text { http://www.ikea.com/ms/de_AT/jobs/join_us/ikea } \\
\text { _values/ }\end{array}$ \\
\hline $\begin{array}{l}\text { Belgium - } \\
\text { French }\end{array}$ & $\begin{array}{l}\text { Travaillez dur, } \\
\text { soyez vous-même }\end{array}$ & $\begin{array}{l}\text { Une culture d'entreprise } \\
\text { basée sur des valeurs } \\
\text { partagées. } \\
\text { Camaraderie } \\
\text { Conscience Des Coûts } \\
\text { Respect } \\
\text { Simplicité }\end{array}$ & $\begin{array}{l}\text { http://www.ikea.com/ms/fr_BE/jobs/join_us/ikea } \\
\text { _values/ }\end{array}$ \\
\hline $\begin{array}{l}\text { Belgium - } \\
\text { Dutch }\end{array}$ & $\begin{array}{l}\text { Werk hard, wees } \\
\text { jezelf }\end{array}$ & $\begin{array}{l}\text { Een bedrijfscultuur op } \\
\text { basis van } \\
\text { gemeenschappelijke } \\
\text { waarden } \\
\text { Samenhorigheid } \\
\text { Kostenbesef } \\
\text { Respect } \\
\text { Eenvoud }\end{array}$ & $\begin{array}{l}\text { http://www.ikea.com/ms/nl_BE/jobs/join_us/ikea } \\
\text { _values/ }\end{array}$ \\
\hline $\begin{array}{l}\text { Canada - } \\
\text { English }\end{array}$ & $\begin{array}{l}\text { Work hard, be } \\
\text { yourself }\end{array}$ & $\begin{array}{l}\text { A corporate culture } \\
\text { based on shared values } \\
\text { Togetherness } \\
\text { Cost-Consciousness } \\
\text { Respect } \\
\text { Simplicity }\end{array}$ & $\begin{array}{l}\text { http://www.ikea.com/ms/en_CA/jobs/join_us/ike } \\
\text { a_values/ }\end{array}$ \\
\hline
\end{tabular}




\begin{tabular}{|c|c|c|c|}
\hline $\begin{array}{l}\text { Canada - } \\
\text { French }\end{array}$ & $\begin{array}{l}\text { Travaillez dur, } \\
\text { soyez vous-même }\end{array}$ & $\begin{array}{l}\text { Une culture d'entreprise } \\
\text { fondée sur des valeurs } \\
\text { communes } \\
\text { Appartenance } \\
\text { Conscience Des Coûts } \\
\text { Respect } \\
\text { Simplicité }\end{array}$ & $\begin{array}{l}\text { http://www.ikea.com/ms/fr_CA/jobs/join_us/ikea } \\
\text { _values/ }\end{array}$ \\
\hline $\begin{array}{l}\text { China- } \\
\text { Chinese } \\
\text { use English } \\
\text { about } \\
\text { values }\end{array}$ & $\begin{array}{l}\text { Work hard, be } \\
\text { yourself }\end{array}$ & $\begin{array}{l}\text { A corporate culture } \\
\text { based on shared values } \\
\text { Togetherness } \\
\text { Cost-Consciousness } \\
\text { Respect } \\
\text { Simplicity }\end{array}$ & $\begin{array}{l}\text { http://www.ikea.com/ms/zh_CN/jobs/join_us/ike } \\
\text { a_values/index.html }\end{array}$ \\
\hline $\begin{array}{l}\text { Czech } \\
\text { Republic }\end{array}$ & $\begin{array}{l}\text { Pracujte tvrdě, } \\
\text { bud'te sami sebou }\end{array}$ & $\begin{array}{l}\text { Podniková kultura } \\
\text { založená na společně } \\
\text { sdílených hodnotách } \\
\text { Soudržnost } \\
\text { Uvědomování Si } \\
\text { Nákladů } \\
\text { Respekt, Úcta } \\
\text { Jednoduchost }\end{array}$ & $\begin{array}{l}\text { http://www.ikea.com/ms/cs_CZ/jobs/join_us/ikea } \\
\text { _values/ }\end{array}$ \\
\hline Denmark & $\begin{array}{l}\text { Arbejd hårdt og vær } \\
\text { dig selv }\end{array}$ & $\begin{array}{l}\text { Virksomhedskultur, der } \\
\text { bygger på fælles } \\
\text { værdier } \\
\text { Samvær } \\
\text { Omkostningsbevidsthed } \\
\text { Respekt } \\
\text { Enkelhed } \\
\text { Åbenhed }\end{array}$ & $\begin{array}{l}\text { http://www.ikea.com/ms/da_DK/jobs/join_us/ike } \\
\text { a_values/ }\end{array}$ \\
\hline Finland & $\begin{array}{l}\text { Työskentele } \\
\text { ahkerasti ja ole oma } \\
\text { itsesi }\end{array}$ & $\begin{array}{l}\text { Yhteisten arvojen } \\
\text { yrityskulttuuri } \\
\text { Yhteenkuuluvuus } \\
\text { Kustannustietoisuus } \\
\text { Kunnioitus } \\
\text { Yksinkertaisuus } \\
\end{array}$ & $\begin{array}{l}\text { http://www.ikea.com/ms/fi_Fl/jobs/join_us/ikea_ } \\
\text { values/ }\end{array}$ \\
\hline France & $\begin{array}{l}\text { Travaillez dur, } \\
\text { soyez vous-même }\end{array}$ & $\begin{array}{l}\text { Une culture d'entreprise } \\
\text { fondée sur des valeurs } \\
\text { communes } \\
\text { Appartenance } \\
\text { Conscience Des Coûts } \\
\text { Respect } \\
\text { Simplicité }\end{array}$ & $\begin{array}{l}\text { http://www.ikea.com/ms/fr_FR/jobs/join_us/ikea } \\
\text { _values/ }\end{array}$ \\
\hline Germany & $\begin{array}{l}\text { Gut arbeiten, sich } \\
\text { treu bleiben }\end{array}$ & $\begin{array}{l}\text { Eine Firmenkultur, die } \\
\text { auf gemeinsamen } \\
\text { Werten aufbaut } \\
\text { Gemeinschaft } \\
\text { Kostenbewusstsein } \\
\text { Respekt } \\
\text { Einfachheit }\end{array}$ & $\begin{array}{l}\text { http://www.ikea.com/ms/de_DE/jobs/join_us/ike } \\
\text { a_values/ }\end{array}$ \\
\hline Hungary & $\begin{array}{l}\text { Dolgozz keményen } \\
\text { és légy önmagad }\end{array}$ & $\begin{array}{l}\text { A Közös Értékeken } \\
\text { Alapuló Vállalati Kultúra }\end{array}$ & $\begin{array}{l}\text { http://www.ikea.com/ms/hu_HU/jobs/join_us/ike } \\
\text { a_values/ }\end{array}$ \\
\hline Italy & $\begin{array}{l}\text { Impegnati e sii te } \\
\text { stesso }\end{array}$ & $\begin{array}{l}\text { Una cultura aziendale } \\
\text { basata su valori } \\
\text { condivisi } \\
\text { Solidarietà } \\
\text { Attenzione Ai Costi } \\
\text { Rispetto } \\
\text { Semplicità }\end{array}$ & $\begin{array}{l}\text { http://www.ikea.com/ms/it_IT/jobs/join_us/ikea_ } \\
\text { values/ }\end{array}$ \\
\hline $\begin{array}{l}\text { Japan - } \\
\text { English }\end{array}$ & $\begin{array}{l}\text { Work hard, be } \\
\text { yourself }\end{array}$ & $\begin{array}{l}\text { A corporate culture } \\
\text { based on shared values } \\
\text { Togetherness } \\
\text { Cost-Consciousness } \\
\text { Respect } \\
\text { Simplicity }\end{array}$ & $\begin{array}{l}\text { http://www.ikea.com/ms/en_JP/jobs/join_us/ikea } \\
\text { _values/index.html }\end{array}$ \\
\hline $\begin{array}{l}\text { Japan - } \\
\text { Japanese }\end{array}$ & $\begin{array}{l}\text { Seems to be similar } \\
\text { to the English } \\
\text { version }\end{array}$ & & $\begin{array}{l}\text { http://www.ikea.com/ms/ja_JP/jobs/join_us/ikea } \\
\text { _values/index.html }\end{array}$ \\
\hline Netherlands & $\begin{array}{l}\text { Werk hard, wees } \\
\text { jezelf }\end{array}$ & $\begin{array}{l}\text { Een bedrijfscultuur die } \\
\text { op gedeelde waarden } \\
\text { rust } \\
\text { Saamhorigheid } \\
\text { Kostenbewustzijn } \\
\text { Respect } \\
\text { Eenvoud }\end{array}$ & $\begin{array}{l}\text { http://www.ikea.com/ms/nl_NL/jobs/join_us/ikea } \\
\text { _values/ }\end{array}$ \\
\hline
\end{tabular}




\begin{tabular}{|c|c|c|c|}
\hline Norway & $\begin{array}{l}\text { Arbeid hardt, vær } \\
\text { deg selv }\end{array}$ & $\begin{array}{l}\text { En forretningskultur } \\
\text { basert på felles verdier } \\
\text { Fellesskap } \\
\text { Kostnadsbevissthet } \\
\text { Respekt } \\
\text { Enkelhet }\end{array}$ & $\begin{array}{l}\text { http://www.ikea.com/ms/no_NO/jobs/join_us/ike } \\
\text { a_values/ }\end{array}$ \\
\hline Poland & $\begin{array}{l}\text { Bądź pracowity, } \\
\text { bądź sobą }\end{array}$ & $\begin{array}{l}\text { Kultura korporacyjna } \\
\text { oparta na wspólnych } \\
\text { wartościach } \\
\text { Poczucie Wspólnoty } \\
\text { Wydajność } \\
\text { Szacunek } \\
\text { Prostota }\end{array}$ & $\begin{array}{l}\text { http://www.ikea.com/ms/pl_PL/jobs/join_us/ikea } \\
\text { _values/ }\end{array}$ \\
\hline Portugal & $\begin{array}{l}\text { Trabalhe muito, seja } \\
\text { você mesmo }\end{array}$ & $\begin{array}{l}\text { Uma cultura corporativa } \\
\text { baseada em valores } \\
\text { partilhados } \\
\text { Espírito De Equipa } \\
\text { Consciência De Custos } \\
\text { Humildade } \\
\text { Simplicidade } \\
\text { Orientação Para O } \\
\text { Cliente }\end{array}$ & $\begin{array}{l}\text { http://www.ikea.com/ms/pt_PT/jobs/join_us/ikea } \\
\text { _values/ }\end{array}$ \\
\hline Russia & $\begin{array}{l}\text { Работай с } \\
\text { максимальной } \\
\text { отдачей, будь } \\
\text { самим собой }\end{array}$ & $\begin{array}{l}\text { Корпоративная } \\
\text { культура } \\
\text { основывается на } \\
\text { определенных } \\
\text { ценностях } \\
\text { Единство } \\
\text { Осознание Расходов } \\
\text { Ответственность } \\
\text { Простота } \\
\end{array}$ & $\begin{array}{l}\text { http://www.ikea.com/ms/ru_RU/jobs/join_us/ikea } \\
\text { _values/ }\end{array}$ \\
\hline Slovakia & $\begin{array}{l}\text { Tvrdo pracujte, } \\
\text { bud'te sám sebo }\end{array}$ & $\begin{array}{l}\text { Firemná kultúra } \\
\text { založená na spoločných } \\
\text { hodnotách } \\
\text { Súdržnost' } \\
\text { Šetrnost' } \\
\text { Úcta } \\
\text { Jednoduchost' }\end{array}$ & $\begin{array}{l}\text { http://www.ikea.com/ms/sk_SK/jobs/join_us/ikea } \\
\text { _values/ }\end{array}$ \\
\hline Spain & $\begin{array}{l}\text { Esfuérzate en el } \\
\text { trabajo, sé tú mismo }\end{array}$ & $\begin{array}{l}\text { Una cultura corporativa } \\
\text { basada en los valores } \\
\text { compartidos } \\
\text { Unión } \\
\text { Consciencia De Costes } \\
\text { Respeto } \\
\text { Sencillez }\end{array}$ & $\begin{array}{l}\text { http://www.ikea.com/ms/es_ES/jobs/join_us/ikea } \\
\text { _values/ }\end{array}$ \\
\hline Sweden & $\begin{array}{l}\text { Arbeta hårt och var } \\
\text { dig själv }\end{array}$ & $\begin{array}{l}\text { En företagskultur } \\
\text { baserad på delade } \\
\text { värderingar } \\
\text { Tillhörighet } \\
\text { Kostnadsmedvetenhet } \\
\text { Respekt } \\
\text { Enkelhet }\end{array}$ & $\begin{array}{l}\text { http://www.ikea.com/ms/sv_SE/jobs/join_us/ikea } \\
\text { _values/ }\end{array}$ \\
\hline $\begin{array}{l}\text { Switzerland } \\
\text { - French }\end{array}$ & $\begin{array}{l}\text { Travaille dur, sois } \\
\text { toi-même }\end{array}$ & $\begin{array}{l}\text { Une culture d'entreprise } \\
\text { basée sur des valeurs } \\
\text { partagées } \\
\text { Camaraderie } \\
\text { Conscience Des Coûts } \\
\text { Respect } \\
\text { Simplicité }\end{array}$ & $\begin{array}{l}\text { http://www.ikea.com/ms/fr_CH/jobs/join_us/ikea } \\
\text { _values/ }\end{array}$ \\
\hline $\begin{array}{l}\text { Switzerland } \\
\text { - German }\end{array}$ & $\begin{array}{l}\text { Arbeite hart, sei du } \\
\text { selbst }\end{array}$ & $\begin{array}{l}\text { Eine Firmenkultur, die } \\
\text { auf gemeinsamen } \\
\text { Werten aufbaut } \\
\text { Gemeinschaft } \\
\text { Kostenbewusstsein } \\
\text { Respekt } \\
\text { Einfachheit }\end{array}$ & $\begin{array}{l}\text { http://www.ikea.com/ms/de_CH/jobs/join_us/ike } \\
\text { a_values/ }\end{array}$ \\
\hline $\begin{array}{l}\text { Switzerland } \\
\text { - Italian }\end{array}$ & $\begin{array}{l}\text { Lavorare sodo, } \\
\text { essere te stesso }\end{array}$ & $\begin{array}{l}\text { Una cultura corporativa } \\
\text { basata su valori } \\
\text { condivisi. } \\
\text { Solidarietà } \\
\text { Consapevolezza Dei } \\
\text { Costi } \\
\text { Rispetto } \\
\text { Semplicità }\end{array}$ & $\begin{array}{l}\text { http://www.ikea.com/ms/it_CH/jobs/join_us/ikea } \\
\text { _values/ }\end{array}$ \\
\hline
\end{tabular}




\begin{tabular}{|l|l|l|l|}
\hline $\begin{array}{l}\text { United } \\
\text { Kingdom }\end{array}$ & $\begin{array}{l}\text { Work hard, be } \\
\text { yourself }\end{array}$ & $\begin{array}{l}\text { A corporate culture } \\
\text { based on shared values } \\
\text { Togetherness } \\
\text { Cost-Consciousness } \\
\text { Respect } \\
\text { Simplicity }\end{array}$ & $\begin{array}{l}\text { http://www.ikea.com/ms/en_GB/jobs/join_us/ike } \\
\text { a_values/ }\end{array}$ \\
\hline $\begin{array}{l}\text { United } \\
\text { States }\end{array}$ & $\begin{array}{l}\text { Work hard, be } \\
\text { yourself }\end{array}$ & $\begin{array}{l}\text { A corporate culture } \\
\text { based on shared values } \\
\text { Togetherness } \\
\text { Cost-Consciousness } \\
\text { Respect } \\
\text { Simplicity }\end{array}$ & $\begin{array}{l}\text { http://www.ikea.com/ms/en_US/jobs/join_us/ike } \\
\text { a_values/ }\end{array}$ \\
\hline $\begin{array}{l}\text { United } \\
\text { States- } \\
\text { Spanish }\end{array}$ & No recruitment page & & http://www.enamoratemasdetuhogar.com/ \\
\hline
\end{tabular}

Table 2: Diversity management in countries run by franchisees outside the IKEA Group

\begin{tabular}{|c|c|c|c|}
\hline Country & $\begin{array}{l}\text { Non-negotiable } \\
\text { differences }\end{array}$ & Negotiable differences & URL \\
\hline Cypruss & No recruitment page & & http://www.ikea.com.cy \\
\hline Greece & No recruitment page & & http://www.ikea.gr/content/index.asp \\
\hline $\begin{array}{l}\text { Hong } \\
\text { Kong - } \\
\text { Chinese }\end{array}$ & Not available page & & http://www.ikea.com.hk/chi/job/job.asp \\
\hline $\begin{array}{l}\text { Hong } \\
\text { Kong - } \\
\text { English }\end{array}$ & & $\begin{array}{l}\text { IKEA has a unique company } \\
\text { culture } \\
\text { We have strong values and } \\
\text { standards that are special to } \\
\text { us - values that create a } \\
\text { sense of belonging. } \\
\text { We offer an environment } \\
\text { where everyone is part of } \\
\text { the team - there are no } \\
\text { barriers between } \\
\text { management and co- } \\
\text { workers and we believe in } \\
\text { leadership by example. }\end{array}$ & http://www.ikea.com.hk/job/job.asp \\
\hline Iceland & No recruitment page & & http://www.ikea.is/ \\
\hline Israel & Not possible to interpret & & http://www.ikea.co.il/ \\
\hline Kuwait & Work hard, be yourself & $\begin{array}{l}\text { A corporate culture based } \\
\text { on shared values } \\
\text { Togetherness } \\
\text { Cost-Consciousness } \\
\text { Respect } \\
\text { Simplicity }\end{array}$ & http://www.ikea.com.kw/Jobatikea.html \\
\hline Malaysia & $\begin{array}{l}\text { The IKEA Human } \\
\text { Resource Idea } \\
\text { Growing with IKEA } \\
\text { At the same time, } \\
\text { everyone is encouraged to } \\
\text { use their initiative - to } \\
\text { question and to try new } \\
\text { ways. Every co-worker is } \\
\text { an important individual to } \\
\text { IKEA. This is why we are } \\
\text { committed to individual } \\
\text { training needs that allow } \\
\text { our people to grow in both } \\
\text { their personal and } \\
\text { professional capacities. }\end{array}$ & $\begin{array}{l}\text { Ikea has a unique company } \\
\text { culture. We have strong } \\
\text { values and standards that } \\
\text { are special to us - values } \\
\text { that create a sense of } \\
\text { belonging. }\end{array}$ & http://www.ikea.com.my/jobs/jobs.asp \\
\hline Romania & No recruitment pages & & http://www.ikea.ro \\
\hline $\begin{array}{l}\text { Saudi } \\
\text { Arabia }\end{array}$ & No recruitment information & & http://www.ikea.com.sa/english/ \\
\hline
\end{tabular}




\begin{tabular}{|c|c|c|c|}
\hline Singapore & $\begin{array}{l}\text { Calling All Singaporeans. } \\
\text { We need more of you. } \\
\text { Growing with IKEA } \\
\text { To give people the } \\
\text { opportunity to grow, both } \\
\text { as individuals and } \\
\text { professionally, so that } \\
\text { together we can commit to } \\
\text { creating a better everyday } \\
\text { life for ourselves and our } \\
\text { customers. Enthusiasm, a } \\
\text { constant desire for } \\
\text { renewal, willingness to } \\
\text { accept and delegate } \\
\text { responsibility, humility and } \\
\text { willpower, simplicity } \\
\text { leadership by example and } \\
\text { daring to be different are } \\
\text { some of our values that } \\
\text { create a greater sense of } \\
\text { belonging and } \\
\text { togetherness. } \\
\text { Every co-worker is an } \\
\text { important individual to } \\
\text { Ikea. This is why we are } \\
\text { committed to individual } \\
\text { training needs that allow } \\
\text { our people to grow in both } \\
\text { their personal and } \\
\text { professional capacities. }\end{array}$ & $\begin{array}{l}\text { IKEA has a unique company } \\
\text { culture }\end{array}$ & http://www.ikea.com.sg/jobs/jobs.asp \\
\hline $\begin{array}{l}\text { Taiwan - } \\
\text { Chinese }\end{array}$ & $\begin{array}{l}\text { Seems to be similar to the } \\
\text { English version }\end{array}$ & & http://www.ikea.com.tw/chi/job/job.asp \\
\hline $\begin{array}{l}\text { Taiwan - } \\
\text { English }\end{array}$ & & $\begin{array}{l}\text { IKEA has a unique company } \\
\text { culture } \\
\text { We have strong values and } \\
\text { standards that are special to } \\
\text { us - values that create a } \\
\text { sense of belonging. }\end{array}$ & http://www.ikea.com.tw/job/job.asp \\
\hline Turkey & & $\begin{array}{l}\text { Togetherness and } \\
\text { enthusiasm } \\
\text { open and honest } \\
\text { communication } \\
\text { modesty } \\
\text { To assume responsibility } \\
\text { and take action } \\
\text { Cost awareness } \\
\text { Concentration and plainness } \\
\text { Leadership through being a } \\
\text { model }\end{array}$ & $\begin{array}{l}\text { http://www.ikea.com.tr/eng/careerparent } \\
\text { _kurumsaldeger.aspx }\end{array}$ \\
\hline $\begin{array}{l}\text { United } \\
\text { Arab } \\
\text { Emirates }\end{array}$ & $\begin{array}{l}\text { Recruitment is done by an } \\
\text { external firm }\end{array}$ & & http://www.ikeadubai.com/ \\
\hline
\end{tabular}

\section{Findings}

The discussion of findings will have two rounds of interpretation. The first round will consider the general differences between the countries and the second round will discuss the tension between statements emphasising individual differences and strong corporate culture.

When we compare the different countries, ownerships stand out to make a difference. Table 1 documents that almost all countries where IKEA owns the stores have similar statements in both categories. Also the URL's are almost similar, which refer to identical web-designs and identical pictures (showing a young woman and a young man, both of Scandinavian origin). It seems to be the case that this recruitment marketing material is controlled and purposely made centrally in the IKEA headquarters. The web-sites from IKEA franchisees outside the IKEA Group are in 
general of a more adaptive character and differ from one another. First, a group of countries (Cyprus, Greece, Iceland, Romania, and Saudi Arabia) do not market job opportunities at IKEA and in United Arab Emirates a link on the web-page brings you to an external recruitment agent. Secondly, the group of countries that has recruitment marketing material on their web-site, the explanation of IKEA values is more detailed and are somewhat adapted to the culture of the country in question. This is the case in Hong Kong, Malaysia, Singapore, Taiwan and Turkey. However, Kuwait stands out in the sense that the statements are similar to the countries where IKEA owns the stores. The findings illustrate that the type of ownerships makes a difference in the sense that full ownerships tends to give a more standardized diversity management policy compared to a more adaptive one when the ownerships is shared as in franchising. Furthermore, it seems to be the case that countries that are relatively more different from the Swedish origin are more careful explaining the corporate culture. This is in line with earlier findings that suggest that the power of the IKEA culture decreases concurrently with the increased distance from Sweden (Björk, 1998).

The second round of the analysis considers all the countries where IKEA owns the stores. Here all the web-pages had similar statements about individual differences and corporate culture. The findings support the speculation made in the introduction that the diversity management practice of IKEA is paradoxical in emphasising at the same time both a strong corporate culture and respect for and leveraging of individual differences. The investigation, however, allows us to go into depth with the nature of the paradox.

Firstly, the statement about individual differences indicates that when working in IKEA you can be yourself and be true to yourself. The specific characteristics that set you apart from the other employees are not seen as problematic and you will not be made to change them. That is as long as you work hard. The combination of "Work hard" and "be yourself" seems to indicate that the acceptance of the selves is conditioned on the self as hard working. So, if you for some reason are not are able to work as hard as your colleagues, you are no longer your-self. Does it mean you have lost your identity?

Secondly, the statements emphasising a strong corporate culture indicate that IKEA has only one, as the statement describes the corporate culture in the singular. The corporate culture is based on shared values. These values are explicated in the other statements as: "togetherness, cost-consciousness, respect and simplicity". There is thus a tension and paradox between on the one hand respecting and leveraging individual differences and on the other enforcing a shared set of values. This is also reflected in statements made by Anders Dahlvig when asked to characterize the leadership of IKEA: "It has to reflect our values, the IKEA culture, and there is a connection between the values and the image the person gives. So if you live the values, you also reflect the culture image. Within those values it is also important for all leaders to develop their own style. We don't try to move them into a specific type of leadership. The framework is our core values, and we allow a lot of freedom depending on who you are and what your specific skills are” (Kling \& Goteman, 2003).

The diversity management of IKEA thus deploys different uses of the concept of difference, which serve different strategic purposes. On the one hand, individual 
differences are respected as non-negotiable. If you are a black woman with a given ethnic background, with the values and characteristics that follow from this, this is respected by IKEA, and even leveraged as we saw earlier: "As a global player it is very important for IKEA to be aware of what the world looks like today, and how it will change in the future. The only common thing for all of us is that we are different. If we accept and understand this fact we can start to use this Diversity for the best of ourselves and for IKEA" (Fajtová, 2007). On the other hand, the individual differences must be aligned with the shared corporate values of IKEA. The very same differences (in so far as they are in conflict with the IKEA values), which were respected and leveraged in first strategic use of difference, are in the second use, negotiated into alignment with corporate values.

This paradox constitutes a contradiction. This contradiction does not, however, constitute an error on the part IKEA. The contradiction is necessary in order for there to be diversity management in IKEA. There must be non-negotiable differences of gender, ethnicity etc. in order for it to make sense, to speak of diversity. At the same time, these differences must be negotiable in order for it to make sense to manage them in a coherent corporation.

Accepting the paradox, whether explicitly or implicitly, opens a field of possibilities, one of which is the practice displayed in the web pages of IKEA. We might inquire, however, if this paradox is specific to the diversity management practice of IKEA.

\section{Differences of difference - towards a theoretical framework for understanding diversity management in international businesses}

Does the theory of diversity management incorporate the paradox described above? One way of finding out is to visit a seminal article in the field. R. Roosevelt Thomas Jr.'s (1990) “From Affirmative Action to Affirming Diversity” is one such article. This article is pivotal in the establishment of diversity management as a field, and as such incorporates many of the central arguments of the field. Among these the notion that differences are a fact of organizational life, and increasingly so, as society at large is becoming increasingly diverse. The task at hand then is to manage these differences in the best possible way. In fact, certain differences in ethnicity and gender can be turned to an advantage. Managing differences has become "a question of business survival” (Roosevelt Thomas Jr., 1990, p. 108). The reason for this is that different people have different competences and talents, and if the management is focused only on white males it will not tap into the talent of minorities.

This potential advantage of differences can be triggered by two mechanisms according to Roosevelt Thomas Jr. The first mechanism relates to the increasingly diverse consumer group of most firms. Minorities may be an increasingly interesting customer group and recruiting minorities as middle managers, for instance, may increase the organization's ability to reach out to these particular customers (Roosevelt Thomas Jr., 1990, p 111). Consequently, the differences within the organization become an advantage as they correlate to the differences in the customer base. This, however, only works if the minorities "bring their ethnicity and/or gender into the organization”. 
The second mechanism relates to an alleged underperformance on the part of the minorities. If an organization can be created where differences are somehow sidestepped, and a new all inclusive "we" can be created, minorities can perform to the same level as adult white males (who constitute the mainstream). This only works if the ethnic and gender differences can be "sidestepped" (Roosevelt Thomas Jr., 1990).

The two mechanisms mentioned above are both found in the IKEA case. The similarity extends also to the two strategic deployments of the term difference. In Thomas Jr.s text differences are either crucial and non-negotiable or to be negotiated and sidestepped, depending on which argument is being made. In the first mechanism minority individuals neither can nor should restrict or limit their difference from mainstream. It is imperative that they maintain the ethnic and/or gender characteristics. In the second, however, the differences must and can be sidestepped to create a new "we". The two different strategies can be termed negotiable and non-negotiable differences. Table 3 gives a few examples of how the two strategies are deployed in statements and arguments in Roosevelt Thomas Jr.'s text.

\section{Table 3: Negotiable and non-negotiable differences in diversity management}

\section{Negotiable differences}

"What we must do is to create an environment where no one is advantaged or disadvantaged, an environment where 'we' is everyone." (109)

“...the organization's leaders must work all the harder to define belonging in terms of values and a sense of purpose that transcend the interests, desires, and preferences of any one group" (116)

In Thomas' examples of organizational crisis, where everybody suddenly pull together as a group to save the organization, the strategy of relativizing differences is also deployed (e.g. p. 114).
Non-negotiable differences

"First, if ever it was possible to melt down Scotsmen and Dutchmen and Frenchmen into an indistinguishable broth, you can't do the same with blacks, Asians and women. Their differences don't melt so easily." (112)

"...the uncomfortable realities of differences" (114)

"Senior management at another corporation saw the growing importance of minorities in their customer base and decided they needed minority participation in their managerial ranks." (111)

There is thus a twofold use of the concept of difference in both the IKEA case and Thomas Jr.s text, and somehow the various uses makes the text and theory function against itself and its own assumptions (Johnson, 2004). One could ascribe this to an error on the author. The two strategic deployments of difference are thus at one and the same time necessary for the establishment of the field, and contradictory; diversity management is inherently contradictory. On the one hand, the relevance and legitimacy rests on non-negotiable differences. On the other hand, only if these differences in some way can be negotiated and sidestepped will diversity management be possible.

We might now be tempted to discard diversity management altogether, as it builds on a contradiction. Another, more constructive, way would be to explore the 
problem in light of the ambiguity of the term difference. The reading of Roosevelt Thomas Jr. demonstrates that differences may at one and the same time be produced and productive. Think of the phrase "make a difference". On the one hand it makes a difference whether you are of European or Arab descendence. This difference is given and non-negotiable. This is why ethnicity is a problem in the first place, but also why it is an advantage to mirror the diversity of your customer base internally within the organization. Differences are thus productive in the sense that they bring about certain states of affairs. We find this notion in the non-negotiable understanding of differences. On the other hand differences are produced. New differences can be produced, as the contextual distinction between the 'we' of the organization against the 'them' of the outside, produced in times of organizational crisis or in IKEA's shared values. But by implication when new differences are produced others are renegotiated. The process of producing a new 'we' of the organization necessarily affects the "older” difference of ethnicity and gender.

The non-negotiable understanding of differences thus emphasizes the productive aspect of differences, while ignoring that differences in themselves are produced. Differences are produced in specific localized settings. That some differences in some cases are perceived as natural, given perhaps by some biological logic is simply a misunderstanding. As the examples given by Roosevelt Thomas Jr. clearly indicate specific local circumstances (a crisis situation in an organization) is quite capable of renegotiating even the most 'natural' differences. The ambiguity of difference and the two (contradictory) deployments of strategic uses of difference thus open a field of possibilities.

\section{Implications for Practice}

The study here shows that the perception of difference in diversity management is paradoxical. The paradox however as shown in the IKEA case opens a field of possible actions. One of these is that it is possible to some extent, and at least in the practice of the web pages, to combine the respect of individual differences with a strong corporate culture. However the case and the following analysis also show that the process of creating and maintaining coherence across an international business, is not necessarily as straightforward as it would seem when looking at the shared values of IKEA. The individual differences which employees bring into the organisation are necessarily negotiated and as such not fixed. Accordingly, the same must necessarily be the case with the differences imposed by the shared values of IKEA. They are always already being negotiated. This means that the shared values are continuously renegotiated in the local setting. The values thus might in some way be shared, but are not the same across national contexts. A strong corporate culture therefore is not the output or effect of the process of creating cohesion. Instead, it may serve as input to local processes of negotiating the individual differences of employees as well as the values of IKEA.

This needs to be considered when managers of international business try to create cohesion across local sections of the company. Shared values are always negotiated in the local context. Consequently, in order for there to be sameness across local contexts the values will be different. 


\section{Conclusion}

This research has shown that the IKEA diversity management practice handles the ambiguity of differences when a thoughtful and reflective diversity management practice is the case. The implications for managers of global firms are that they need to recognise and handle the embedded paradox in diversity management as a management tool to handle the internationalisation process of the firm, where the globalization myth that everything is the same is rejected and at the same time is maintained when utilising diversity management.

Several issues related to this conclusion calls for more research. Firstly, we have not analyzed the different (and similar) images on the web-pages, which could make a stronger and more nuanced analysis. This explorative study should be accompanied with interviews of central actors as manager from the IKEA headquarters and local co-workers in order to challenges the understanding of IKEA's diversity practice. Cross-national surveys would be useful to challenge the understandings of the non-negotiable differences and the negotiable differences.

\section{References}

Ayal, I., \& Zif, J. 1979. Market Expansion Strategies in Multinational Marketing. Journal of Marketing, 43(2).

Björk, S. 1998. IKEA. Ingvar Kamprad og hans imperium. København: Børsen.

Capell, K. 2006. Ikea's New Plan for Japan, BusinessWeek.

Capell, K., Sains, A., Lindblad, C., Palmer, A. T., Bush, J., Roberts, D., \& Hall, K. 2005. Ikea - How the Swedish Retailer became a global cult brand BusinessWeek.

Cox, T. H., \& Blake, S. 1991. Managing cultural diversity: Implications for organizational competitiveness. The Executive, 5(3): 45-58.

Datamonitor. 2005. Denmark: Key Facts and Overview: Datamonitor plc.

Douglas, S. P., \& Craig, C. S. 1989. Evolution of Global Marketing Strategy: Scale, Scope and Synergy. Columbia Journal of World Business, 24(3): 47-59.

Fajtová, E. 2007. Why Diversity? A global experience, Conference: Diversity Management \& Inclusion: European Solutions to Common Problems. Prague, The Czech Republic: Human Resources Development Interface.

Grol, P., \& Schoch, C. 1998. IKEA: Managing Cultural Diversity. In G. R. Oddou, \& M. A. Mendenhall (Eds.), Cases in international organizational behavior. Malden, Ma.; Oxford, England: Blackwell Publishers.

Guillen, M. F. 2001. Is Globalization Civilizing, Destructive or Feeble? A Critique of Five Key Debates in the Social Science Literature. Annual Review of Sociology, 27: 235-260.

Hollensen, S. 2007a. Global Marketing - A decision-oriented approach (4 ed.). Essex, England: Financial Times Press.

Hollensen, S. 2007b. IKEA: Expanding through franchising to the South American market? - Case Study III.1, Global Marketing - A decision-oriented approach, 4 ed. Essex, England: Financial Times Press.

IKEA. 2006. why work at IKEA?, Vol. 2007: Inter IKEA Systems B.V.

IKEA. 2007a. Facts \& Figures, IKEA Group 2007: Corporate PR, IKEA Services AB.

IKEA. 2007b. IKEA Group stores, Vol. 2007: Inter IKEA Systems B.V. 
Johnson, B. 2004. Translator's Introduction. In J. Derrida (Ed.), Dissemination: viiXxxv. London: Continuum Books.

Kling, K., \& Goteman, I. 2003. IKEA CEO Anders Dahlvig on international growth and IKEA's unique corporate culture and brand identity. The Academy of Management Executive, 17(1): 31-37.

Kochan, T., Bezrukova, K., Ely, R., Jackson, S., Joshi, A., Jehn, K., Leonard, J., Levine, D., \& Thomas, D. 2002. The Effect of Diversity on Business Performance:: Diversity Research Network.

Krugman, P. 1990. A model of Balance of payments Crises. Journal of Money, Credit and Banking, 11(August): 311-325.

Perlmutter, H. V. 1969. The tortuos evolution of the multinational corporation. Columbia Journal of World Business, 4(Jan-Feb).

Roosevelt Thomas Jr., R. 1990. From Affirmative Action to Affirming Diversity. Harvard Business Review, 68(2): 107-117.

Solomon, B. 1991. A Swedish Company corners the Business: Worldwide. Management Review, 80(4): 10-14.

Thomas, D. A., \& Ely, R. J. 1996. Making difference matter: A new paradigm for managing diversity. Harvard Business Review, Sep-Oct: 79-90.

Woods, R., \& Sciarini, M. 1995. Diversity programs in chain restaurants. Cornell Hotel and Restaurant Quartely, 36(3): 18-23.

Wrench, J. 2002. A Critical Analysis of Critiques of Diversity Management. Paper presented at the Seventh International Metropolis Conference -Togetherness in Difference: Citizenship and Belonging, Oslo.

Yip, G. S. 1989. Global Strategy...In a World of Nations? Sloan Management Review, 31(1): 29-42.

Zachary, L. J. 2005. Creating a mentoring culture: the organization's guide (1st ed.). San Francisco: Jossey-Bass. 